\title{
EDITORIAL
}

\section{Presentation of data}

One of the questions that we ask of editors and referees concerns the suitability of and need for the tables and figures that authors submit with their papers. The layout of tables and the quality of figures submitted for publication is frequently a cause of great concern to the staff of the Editorial Office, especially those who have to do the technical editing of papers, and is often a cause of delay in processing papers because either tables or figures have not been prepared according to the 'Directions to Contributors' and they have to be returned for retyping or redrawing.

The style of tables and the standard of published figures are important for the appearance of the Journal and also have been chosen for their clarity in conveying information to the readers. We have to insist on these requirements for submitted papers because of the difficulties that improperly prepared tables or figures create during technical editing and publication.

Authors are increasingly submitting computer-generated figures and, while it is possible to prepare figures of the highest standard in this way, many figures are submitted in a form that is not adequate for publication because the contrast and definition are inadequate or the lines are not continuous. Authors should look at the figures submitted for publication very carefully and especially consider what they will look like when they are reduced, as most figures will be in the published paper.

Leaving aside these technical points there are several more important scientific issues regarding the presentation of data that I would like to discuss. These concern the need for authors to consider more carefully the presentation of data in their papers and whether the chosen mode is the best way of communicating the information to the readers.

It is important to see the tables and figures as an integral part of the paper. Although we expect tables and figures to be capable of comprehension in themselves without reference to the text, they should also contribute to the exposition of the experimental results and justify and support the scientific arguments and conclusions being drawn.

There is an understandable wish by many authors to present all the data they have acquired during the work being reported, whether they are relevant or not to the points being made in the text. We receive exquisitely complex tables, sometimes too large to be accommodated within the confines of the page of the Journal, and littered with symbols for footnotes and indicators of statistical significance. Very often one then finds a statement in the text that 'table $\mathrm{n}$ shows...', and so it does, if you spend several hours deciphering the table and its symbols and complexities. In many cases the author could communicate far more effectively through a few smaller tables presenting the data more selectively. In other papers authors insist on taking the reader through all the data in a table, as if the reader were incapable of reading numbers, when a more selective textual commentary would be more effective and, incidentally, make much more interesting reading by focusing attention on those data that really matter.

I recognize the value of making experimental data available in detail to all those working in the field and of creating archival records of original data. My own researches have benefited by being able to read the older literature where authors such as Atwater and Rubner had the opportunity denied to present-day workers of publishing all their original observations. I think that we need to consider very carefully how such original material can 
be made more available. This is particularly important for many nutritional studies such as epidemiological ones where food intakes have been measured because the methodologies followed in such work are critically important to the interpretation of the results, especially where structured questionnaires such as food frequency questionnaires have been used. Another area where it is often difficult to persuade editors that original data are worth publishing is that of food or dietary composition. Clearly there is a need for implementing some kind of nutritional sciences archive.

The choice of expressing data in tables or figures is sometimes difficult and I occasionally get the impression that a random selection has been made and, less frequently, I feel that a figure has been used because the author does not want either our statistical editors or the readers to look too closely at the actual figures. I must declare a preference for tables of data and these are more useful for proper statistical appraisal.

I accept that figures are extremely useful when showing time trends and when discussing patterns of results. As a general rule the British Journal of Nutrition is not inclined to have the same data in both tables and figures, but figures may illustrate a point in the discussion more effectively than referring the reader back to tables. It really is up to the author to persuade the editors that the scientific points that the paper is making are improved by the use of both tables and figures. This means that the author must think very carefully about integrating the presentation of data into the paper as an entity.

When the Editorial Report includes a comment such as 'the authors should reconsider the tables or figures in the paper' this is often a coded message that we believe that the data in the paper could be presented more effectively, and as a general rule most papers would be improved if more attention were given to considering how best to present the data as an integral part of the paper.

D. A. T. Southgate 